\title{
Surveillance case definitions for work related upper limb pain syndromes
}

\author{
J M Harrington, J T Carter, L Birrell, D Gompertz
}

\begin{abstract}
Objectives-To establish consensus case definitions for several common work related upper limb pain syndromes for use in surveillance or studies of the aetiology of these conditions.

Methods-A group of healthcare professionals from the disciplines interested in the prevention and management of upper limb disorders were recruited for a Delphi exercise. A questionnaire was used to establish case definitions from the participants, followed by a consensus conference involving the core group of 29 people. The draft conclusions were recirculated for review.

Results-Consensus case definitions were agreed for carpal tunnel syndrome, tenosynovitis of the wrist, de Quervain's disease of the wrist, epicondylitis, shoulder capsulitis (frozen shoulder), and shoulder tendonitis. The consensus group also identified a condition defined as "non-specific diffuse forearm pain" although this is essentially a diagnosis made by exclusion. The group did not have enough experience of the thoracic outlet syndrome to make recommendations.

Conclusions-There was enough consensus between several health professionals from different disciplines to establish case definitions suitable for use in the studies of several work related upper limb pain syndromes. The use of these criteria should allow comparability between studies and centres and facilitate research in this field. The criteria may also be useful in surveillance programmes and as aids to case management.
\end{abstract}

(Occup Environ Med 1998;55:264-271)

Keywords: upper limb pain; occupational; epidemiology

Institute of

Occupational Health,

University of

Birmingham,

Birmingham, UK

J M Harrington

J T Carter

L Birrell

D Gompertz

Correspondence to: Professor J M Harrington, Institute of Occupational Health, University of

Birmingham, PO Box 363, University Road West,

Birmingham B15 2TT, UK.

Accepted 4 October 1997 skeletal disorders are caused or made worse by work. ${ }^{2}$ A further 74000 complained of repetitive strain injury (RSI) with most cases confined to the upper limb and most of those to the hand, wrist, and forearm.

Although there is little doubt that the relation to repetitive work or sustained posture is biologically plausible for some of these conditions, considerable confusion and controversy surrounds all aspects of work related upper limb disorders. ${ }^{3}$ Many of the better designed studies fall well short of providing conclusive evidence of a causal relation between work practice and upper limb disorder. ${ }^{4}$ There is difficulty in comparing the various studies that have been designed to investigate the prevalence of these conditions and the role of biomechanical and psychosocial factors in their aetiology. This is partly due to the lack of agreed case definitions and diagnostic criteria for the various upper limb syndromes. 5

The development of diagnostic criteria usually proceeds through a series of well defined stages. Investigators often establish inclusion and exclusion criteria with classic textbook definitions. As a range of clinical or laboratory tests are developed these can be incorporated into the criteria to be used and investigated for their reliability and reproducibility. Formal approaches for establishing the specificity, sensitivity, and predictive value of individual tests have been described and their usefulness established by those techniques used in evidence based medicine. ${ }^{6}$ Difficulties occur, as in the case of work related upper limb pain syndromes, when in the absence of agreed case definitions, each set of research workers use different criteria which are defined to a greater or lesser extent in the reporting of their studies. A particularly pertinent example of this was noted in an attempt at meta-analysis of shoulder-neck disease prevalence in different occupational groups. ${ }^{7}$ The authors noted the dearth of longitudinal studies and the absence of comparable study criteria even for age, exposure, or effect. This is the situation for most work related upper limb disorders; however, the criteria developed in the United States for carpal tunnel syndrome (CTS) are an exception. ${ }^{8}$ For other work related upper limb disorders, there is not enough information available to establish case definitions based on studies of the reproducibility, specificity, sensitivity, and predictive value of individual clinical or laboratory tests. For many the underlying lesion has not been defined, nor is there a gold standard against which to measure the performance of a test. ${ }^{9}$ 
In the absence of appropriate data, a first stage in development of case definitions for work related upper limb disorders could be to capture the experience of expert practitioners in the diagnosis and treatment of these conditions. After such an exercise and if a consensus were established, then formal studies of the diagnostic performance of the newly agreed criteria could be developed. ${ }^{6}$ Such an approach has worked successfully for hand-arm vibration syndrome after the Stockholm classification meetings. ${ }^{10}$ There are several formal methods for collecting and amalgamating the experience and judgement of experts; one often used is the Delphi technique. ${ }^{11}$ Here we report the use of a Delphi exercise to establish case definitions for several clinical conditions of the upper limb associated with work.

\section{Methods}

The development of consensus case definitions was a three stage process based on the Delphi technique. This technique is a group process aimed at developing agreed views by collating, analysing, and then re-presenting structured information about a topic, with a group of experts. ${ }^{11}$ The technique is currently being used to capture professional judgement in a range of biomedical, life, and engineering sciences. In health care it can be used to develop baseline probabilities for diagnosis and care planning in situations in which a gold standard of diagnostic accuracy — such as a specific pathological change-is not available or cannot ethically be obtained. ${ }^{6}$ However, the validity of the Delphi technique can be questioned and this is considered further in the discussion section. The first stage of the present study was to establish by postal questionnaire the range of case definitions in current use for several of the commoner conditions.

Nine upper limb conditions were selected for the study. The selection was based on reviews prepared in 1996 for the United Kingdom Health and Safety Executive by Silman and Helliwell (unpublished) and on an informed view of their frequency. The conditions selected were initially divided into two groups: (a) carpal tunnel syndrome, tenosynovitis (wrist), pain syndrome of forearm or hand, lateral epicondylitis, frozen shoulder; (b) De Quervain's tenosynovitis, shoulder tendonitis, shoulder capsulitis, thoracic outlet syndrome.

A core group of participants who would take part in the Delphi exercise and the subsequent consensus workshop was established, representing the range of disciplines interested in prevention and management of upper limb disorders arising from work. This core group of 29 United Kingdom experts came from the following disciplines, rheumatology (six), surgery (three), occupational health (eight), epidemiology (three), general practice (one), physiotherapy (two), ergonomics (three), psychiatry and psychology (two), and pain physiology (one). The participants were chosen for various reasons. The main one was active involvement in either the clinical management or the epidemiological investigation of work related upper limb disorders. Two neurologists were invited to join the group but as their main contribution would be for the discussion of CTS, both suggested that a neurophysiologist would be more appropriate. A leading member of this specialty agreed to participate. All these core participants were asked to complete proformas on the five conditions in group $a$ and were given the option of completing them on the others in group $b$. They were also asked to indicate any other upper limb disorder that should be considered. Carpal tunnel syndrome was selected as a reference condition on which responses for the others could be modelled as it is clinically the most well defined and a surveillance case definition had already been published in the United States. $^{8}$

The proforma for each condition was structured to ask the participants to give a definition, major and minor diagnostic criteria, any relevant comments, and their professional affiliation. The participants were asked not to include work relatedness either in the definition or criteria for diagnosis. They were sent three sets of proformas and asked to recruit two colleagues they considered able to give additional informed opinions.

The results from this process were then collated for review at a consensus workshop (Birmingham, February 1997) at which formal case definitions for surveillance purposes were to be agreed where possible. The results of the first stage of the Delphi exercise for each condition were presented to the workshop participants as the percentage of respondents listing individual diagnostic features ranked by frequency of responses. (For example, if 25 of 50 respondents listed pain as a characteristic feature, then the percentage was $50 \%$.) Discussion followed on the way in which the condition could be defined and on the combination of criteria required for case definition. An agreed version for each condition was recorded at the end of each session. The Delphi process was completed by sending the draft report of the workshop to the core participants so that they might have an opportunity upon reflection, to review the draft case definitions.

\section{Results}

The proforma exercise generated a total of 340 individual responses, with the number for each condition varying from 45 for carpal tunnel syndrome to 26 for shoulder capsulitis. Most respondents considered shoulder capsulitis and frozen shoulder to be manifestations of the same condition and the two were amalgamated for discussion at the workshop. The analyses of the proforma responses and consensus views of the case definition criteria for each condition are presented here.

\section{CARPAL TUNNEL SYNDROME}

The proforma exercise showed that there was general agreement on the main features and diagnostic techniques but the emphasis varied considerably and reflected the situations in which different groups of participants saw the condition and the decisions they took based on 
Table 1 Percentage of questionnaire responses $(n=45)$ to carpal tunnel syndrome

\begin{tabular}{ll} 
Sensory symptoms-median nerve distribution & 91 \\
Abnormal nerve conduction tests & 80 \\
Phalen's test positive & 50 \\
Tinel's test positive & 43 \\
Weakness or wasting & 41 \\
Sensory loss & 39 \\
Nocturnal exacerbation & 25 \\
\hline
\end{tabular}

their diagnoses. The definition of the condition was not controversial (table 1).

Some respondents included the so called "flick test" as a diagnostic indicator. This refers to reporting by patients of relief from a rapid flick of the hand. Other respondents included relief after corticosteroid injection into the carpal tunnel and reduced grip strength as useful indicators.

This condition is far more thoroughly characterised and investigated than the others considered and this influenced the discussion. For instance the validity of some of the diagnostic tests has been measured and a surveillance case definition had been published in the United States to standardise arrangements for reporting the disease. ${ }^{8}$

The distribution of pain to the area supplied by the median nerve is an important clinical feature but one which is unlikely to be noted by patients. Pain may also be experienced in the forearm up to the elbow. The sparing of the dorsum of the hand and little finger has to be sought by the examiner. This may be simpler for the objective tests of sensation than for pain. Such negative findings can be important in making the diagnosis. Motor loss and wasting of the abductor pollicis brevis are late signs. The extent of the distribution of symptoms such as pain or paraesthesia were not defined, although these would need to be described in any surveillance study.

The role of simple clinical tests such as Phalen's and Tinel's tests in diagnosis was a matter for some discussion. Both tests require standardisation. The sensitivity and specificity of both have been assessed; neither rate very highly but Phalen's test is rather more specific. Some participants considered Tinel's sign to be virtually worthless.

The role of nerve conduction studies has been controversial. ${ }^{12}$ However, it was agreed that when they are available, they are a useful source of supporting information to confirm focal median nerve compression. It was noted that normative values vary between centres and populations and thus local comparison groups are needed for studies. There are some problems with sensitivity and specificity; about $10 \%$ of subjects with a good history of carpal tunnel syndrome will have normal nerve conduction studies (and a proportion of these will respond symptomatically to carpal tunnel decompression). ${ }^{13}$ It seems that subclinical median nerve compression at the wrist is not uncommon in patients referred for other reasons. In the absence of a gold standard test for carpal tunnel syndrome, nerve conduction studies do provide objective evidence of median nerve compression and this is the best test compared with elicited clinical signs - such

as Phalen's or Tinel's sign. (The absence of a neurologist from the group may have influenced the outcome as these clinicians are reported to be the most accurate in diagnosing CTS. $)^{14}$

\section{Consensus}

Agreements were reached on both definition and the surveillance criteria;

Definition-A clinical syndrome caused by compression of the median nerve as it passes through the carpal tunnel

Surveillance criteria-Pain, or paraesthesia, or sensory loss in the median nerve distribution and one of: Tinel's test positive, Phalen's test positive, nocturnal exacerbation of symptoms, motor loss with wasting of abductor pollicis brevis, and abnormal nerve conduction time.

Additional features that might support the diagnosis are: no signs or symptoms in the little finger and on the dorsum of the hand, no other cause apparent, history of successful steroid injection or surgery.

\section{TENOSYNOVITIS OF THE WRIST}

The questionnaire responses showed general agreement with varying emphasis being given to the exclusion of other conditions, especially de Quervain's disease (table 2). It was emphasised that the site of inflammation was of importance; tenosynovitis is a disease of tendon sheaths and the diagnosis should be restricted to this.

In discussion the participants agreed that the key symptom is pain while the wrist is being used, localised to the relevant tendon sheaths. The pain is reproduced by resisted active movement and may also be reproduced by passive stretch and isometric force. Pain may be only experienced during one direction of movement and may also be felt over parts of the tendon which do not have a sheath. The extensor tendon sheaths are the usual site, although a similar condition can occur in the flexors. The relative contribution of inflammation, trauma, and other factors in leading to the condition is unclear.

It was noted that crepitus in the sheath is often only transient. The localised condition of peritendonitis crepitans in the forearm is a separate entity. The tuck sign, in which the tendon sheath bunches up on the dorsum of the hand during finger extension, is an additional indicator of the condition.

Table 2 Percentage of questionnaire responses $(n=41)$ to tenosynovitis of the wrist

Inflammation
Pain (all types)
Pain (specified movement):
Resisted active
Passive stretch
Usage
Rest
Crepitus
Swelling
Tenderness
Erythema
Weakness
Warmth
Reduced function
Reduced movement


Consensus

Definition-Inflammation of the extensor or flexor tendon sheaths at the wrist.

Surveillance criteria-Pain on movement localised to the affected tendon sheaths in the wrist and reproduction of pain by resisted active movement of the affected tendons with the forearm stabilised.

There are additional features that would give greater confidence in diagnosis-namely, history of crepitus, tenderness, or swelling over affected tendon sheaths - but the evaluation of these for surveillance purposes requires further work.

DE QUERVAIN'S DISEASE OF THE WRIST

There was general agreement on the nature and diagnosis of this condition. Many respondents favoured describing it as a tenovaginitis and pointed to the inappropriateness of the term tenosynovitis for a condition in which the synovial tendon sheath is not inflamed (table 3).

Table 3 Percentage of questionnaire responses $(n=36)$ to de Quervain's disease of the wrist

\begin{tabular}{lr}
\hline Pain or tenderness over radial styloid & 78 \\
Exacerbated by thumb extension & 56 \\
Finkelstein's test positive & 64 \\
Swelling or thickening of 1st extensor & \\
$\quad$ compartment & 64 \\
Crepitus & 17 \\
Pain on thumb adduction & 5 \\
Triggering & 5 \\
\hline
\end{tabular}

The pathology of this condition is not well established but it was agreed that there is no evidence of an inflammatory process. It is not clear whether the constriction of tendons derives from changes in the first extensor compartment or from the tendons within it. The key symptom was considered to be pain which is exacerbated by resisted thumb extension. This can be assessed with Finkelstein's test.

In practical terms, this condition needs to be distinguished from osteoarthritis of the wrist or first carpometaphalangeal joint, wrist ligament strains, and scaphoid non-union. Those participants with case management experience agreed that although some cases recover spontaneously, others are only relieved by corticosteroid injection or surgical decompression. These issues were not discussed further.

Consensus

Definition-Painful swelling of the first extensor compartment containing extensor pollicis brevis and adductor pollicis longus.

Surveillance criteria-Pain which is centred over the radial styloid and tender swelling of first extensor compartment and either pain reproduced by resisted thumb extension or positive Finkelstein's test.

NON-SPECIFIC DIFFUSE FOREARM PAIN

The responses to the questionnaire showed that there was a wide range of views on whether and how this condition should be character-
Table 4 Percentage of questionnaire responses $(n=31)$ to non-specific diffuse forearm pain

Pain in forearm

Absence of other causes

Tender muscles

Reduced grip strength

Unpleasant sensations

Related to work

Functional loss

ised. The lower response than for carpal tunnel syndrome was not a consequence of any one professional group not responding (table 4).

There was considerable debate on the terminology with the participants concerned about the controversy associated with this condition and the need not to add further confusion. It was agreed that diffuse forearm pain not associated with any specific anatomical structure may well be a real entity and it is certainly associated with considerable morbidity. However, categorisation of a disease solely based on such pain and on a predisposing pattern of activity was not favoured at this stage. The use of the term repetitive strain injury for this condition or others was considered to be unhelpful. Descriptions of work related conditions have often included activity as an essential prerequisite for the diagnosis. However, in studies of forearm pain, activity is likely to be one of the variables to be studied and so it cannot usefully form a criterion for case definition.

A common view was that this was a diagnosis made by exclusion and this influenced the way in which criteria were framed. The need for better characterisation was recognised and possible subdivision into several conditions with more readily identifiable features suggested. The question of whether this was a syndrome - that is, a constellation of associated features-or whether it was simply a term for those cases of limb pain which could not otherwise be classified was unresolved. Indeed, pain in the forearm was the main conclusion from 45 minutes discussion! As well as the main feature of pain there are some associated aspects which can be assessed. Thus the speed of fine movement can be measured with the Wigley test, in which the time to touch all fingers with the thumb four times in rapid sequence is measured and compared with the unaffected side. Questioning can elicit a range of symptoms: aching, tiredness, cramp, weakness, tremor, loss of function, numbness, paraesthesia, allodynia, and subjective feeling of swelling. Differential diagnosis is from multiple regional pain syndromes, referred pain, and other more specific forearm conditions.

It was agreed that studies of the frequency of relevant symptoms in groups with differing patterns of upper limb activity are needed to establish whether a distinct forearm pain syndrome exists. In principle this is no different from characterising non-specific low back pain or headache as clinical entities.

\section{Consensus}

Definition-Pain in the forearm in the absence of a specific diagnosis or pathology. 
Surveillance criteria-Pain in the forearm and failure to meet the diagnostic criteria for other specific diagnoses and diseases.

Other features which may be present but which are not specific defining criteria include: loss of function, weakness, cramp, muscle tenderness, allodynia, and slowing of fine movements.

\section{LATERAL EPICONDYLITIS}

There was a large measure of agreement on the signs and symptoms of the condition but a diversity of views on the role of inflammation, trauma, and other factors in its cause (table 5). The term epicondylitis was thought to be a misnomer. Tennis elbow was not considered to be a good description as it neither identified a common cause nor characterised the lesion.

Table 5 Percentage of questionnaire responses $(n=42)$ to lateral epicondylitis

\begin{tabular}{ll}
\hline Epicondylar pain & 76 \\
Pain on resisted extension & 80 \\
Epicondylar tenderness & 73 \\
\hline
\end{tabular}

The main symptom is epicondylar pain which often radiates down the arm. It was noted that the precise location of tenderness is usually about $1 \mathrm{~cm}$ distal to the epicondyle. The pain is associated solely with active and resisted movements of the extensor muscles of the forearm.

The pathology of the condition is unclear. Where biopsy has been performed there have been few signs of inflammation, with degenerative appearances predominating. Frank tears at the common extensor origin are rare. Differential diagnosis is from referred neck pain, posterior interosseous nerve entrapment, radial tunnel syndrome, fibromyalgia, and systemic arthropathies. Epicondylitis can develop quickly; the duration of the condition is variable and its natural history has not been thoroughly investigated.

It was agreed that the same considerations applied to medial epicondylitis (golfer's elbow), with corresponding effects on the forearm flexors.

Consensus

Definition-A lesion at the common extensor origin of the lateral epicondyle of the humerus causing the effects noted in the criteria.

Surveillance criteria-Lateral epicondylar pain and epicondylar tenderness and pain on resisted extension of the wrist.

Similar criteria apply to medial epicondylitis and with pain on resisted flexion of the wrist.

SHOULDER CAPSULITIS (FROZEN SHOULDER)

Most respondents considered shoulder capsulitis and frozen shoulder to be the same condition, hence the two were amalgamated for discussion at the workshop. The participating surgeons tended to define the condition more closely in terms of observable adhesions rather than solely as a functional deficit. When respondents did distinguish between the condi-
Table 6 Percentage of responses $(n=42)$ to shoulder capsulitis (frozen shoulder)

Restricted movement (active and passive) Pain at shoulder

Characteristic time course

Reduced synovial volume (arthroscopy or MRI)

Joint tenderness

No radiological abnormality

History of triggering event or condition

tions a broadly similar pattern of features was identified. The data presented are for frozen shoulder as there were more replies on this condition (table 6).

This was the one condition discussed where there was a well developed view on the evolution of signs and symptoms; with the painful initial stage of capsulitis leading to global limitation of movement causing a frozen shoulder, eventually followed by resolution and return of full or partial function. The condition is almost always unilateral although it is recognised that there is an increased risk of developing it subsequently in the other shoulder $(17 \%$ over five years).

The symptoms were not controversial. Restriction of movement was both active and passive, with pain as the limiting factor mainly in the early stages. The restriction is a consequence of glenohumeral rather than acromioclavicular limitation. Limitation of movements has a characteristic pattern: external rotation $>$ abduction $>$ internal rotation. Pain is poorly localised and may radiate widely. It is usually maximal over the lateral aspect of the upper arm (deltoid area). It may be mainly related to movement or can be worst at night. The diagnostic use of arthroscopy and MRI to identify reduction in synovial cavity volume was noted. Differential diagnosis is from radicular neck pain, referred cardiac pain, and glenohumeral arthropathies. The condition itself can be a sequel to other diseases such as pulmonary disorders and cardiovascular disease as well as to injuries and immobilisation of the neck and shoulder.

As well as surveillance criteria the development of an agreed staging system and measures of severity would be useful investigative and clinical tools.

\section{Consensus}

Definition-A condition characterised by current or past pain in the upper arm, with global restriction of glenohumeral movement in a capsular pattern.

Surveillance criteria-History of unilateral pain in the deltoid area and equal restriction of active and passive glenohumeral movement in a capsular pattern (external rotation $>$ abduction $>$ internal rotation).

\section{SHOULDER TENDONITIS}

There was general agreement on the features of the condition but some saw it as a single diagnostic entity whereas others favoured the identification of four separate case definitions based on the tendon affected. Some but not all respondents included bicipital tendonitis in 
Table 7 Percentage of questionnaire responses $(n=30)$ to shoulder tendonitis

\begin{tabular}{ll}
\hline Shoulder pain (all forms) & 90 \\
Pain specified: & 76 \\
Pain on resisted active movement & 47 \\
Pain on abduction, supraspinatus & \\
Pain on external rotation, infraspinatus or teres & 20 \\
minor & 20 \\
Pain on internal rotation, subscapularis & 43 \\
Tenderness & 17 \\
Impingement &
\end{tabular}

their definition hence the summary figures do not cover this condition (table 7).

Views varied on whether this was usefully considered as a single condition when the tendons from one or more of five muscles could be involved, each with different features and in the case of the biceps, with pronounced differences in signs and symptoms from the tendons forming the rotator cuff.

Clinical features of rotator cuff tendonitis may include: a painful arc which is more apparent on active than on passive movement, pain which is worse at night, crepitus, subacromial tenderness, referred pain along C5 distribution, and impingement syndrome-pain and restriction of abduction beyond $80^{\circ}$.

Two named tests for biceps tendonitis are: Speed's test-flexion of elbow against resistance produces pain over the long head of biceps tendon; and Yergason's test-pain over the long head of the biceps tendon on resisted supination of the forearm with elbow flexed to $90^{\circ}$.

There may be both degenerative and inflammatory elements in the condition. Calcification on radiography is taken as evidence of old injury or degeneration. Appearances on MRI can suggest inflammation. The differential diagnoses are glenohumeral capsular disease, acromioclavicular osteoarthritis, and referred cervical, pleural, or cardiac pain.

\section{Consensus}

Definition-Symptomatic inflammation or degeneration of the tendons of the rotator cuff or biceps.

Surveillance criteria-Rotator cuff: history of pain in the deltoid region and pain on one or more resisted active movements (abduction of the supraspinatus; external rotation of the infraspinatus, teres minor; internal rotation of the subscapularis). Biceps: history of anterior shoulder pain and pain on resisted active flexion of elbow or supination of forearm.

THORACIC OUTLET SYNDROME

There was general agreement on the definition and on signs and symptoms. Diagnostic tests were controversial. The role of a cervical rib or band as the cause of the syndrome was disputed. The condition was very rare in the experience of respondents and although symptoms may be exacerbated by work it is probably not initiated by work.

Two sets of features were recognised as neurogenic-pain, sensory or motor deficit attributable to the lower trunk of the brachial plexus - and vascular-arterial or venous insufficiency leading to peripheral signs (table 8).
Table 8 Percentage of of questionnaire responses $(n=33)$ to thoracic outlet syndrome

Neurological abnormalities, ulnar distribution

Reduced blood flow, various signs

Cervical rib or band

Nerve conduction defects

Wasting of hand muscles

Abnormal vascular imagin

Raynauds, ischaemia, embolism

Symptoms from specific movements or postures
Subclavian bruit

Few workshop participants had clinical experience of this condition, hence most Delphi responses were based on the reports of others. This rarity and lack of experience meant that the group was not well placed to specify case definition criteria.

A wide range of symptoms are reported and these can be characterised depending on whether an artery, vein, or nerve is obstructed. Fibrous bands rather than cervical ribs were considered to be the usual cause. There is considerable variation in effects between people and a single set of classification criteria are unlikely to be definable.

The greater frequency of this diagnosis in the United States was noted and may be related to differences in diagnostic conventions and in indications for surgical treatment. There are several diffuse shoulder-neck syndromes, reported particularly in sedentary office populations which may have features which overlap with thoracic outlet syndrome but which lack the specific features or evidence of an obstructive cause. These were not considered. The participants did not consider that this condition was attributable to work and they were not well placed to improve on textbook descriptions.

\section{Consensus}

Definition-A constellation of symptoms and signs in the arm or hand caused by compression of the neurovascular bundle at the thoracic outlet.

Surveillance criteria-None formulated. This was considered to be a very rare condition in United Kingdom practice.

\section{Discussion}

The objectives of this study were, for a selected number of upper limb pain syndromes, to establish case surveillance criteria that could be used in future studies of their prevalence and aetiology. A group of acknowledged experts from the professions responsible for the monitoring, diagnosis, and treatment of upper limb disorders in the United Kingdom was recruited for this exercise. Both the questionnaire and consensus conference stages showed that considerable agreement was possible. Attempts to analyse the response by the professional group of the respondents showed no consistent evidence of any systematic differences (notwithstanding the absence of a neurologist for the CTS discussion).

This Delphi exercise concentrated on the diagnosis of individual conditions for the purpose of prevalence studies and aetiological 
investigations. The overall process of sequentially applying diagnostic methods to establish the best fit for a set of signs and symptoms was not considered. Neither was the extent and distribution of symptoms clearly defined in all conditions considered. During the consensus meeting it became clear that a means of excluding systemic disorders of the musculoskeletal system is needed when diagnosing work related biomechanical disorders. The GALS locomotor screen was considered a useful screening tool. ${ }^{15}$

There is little information on the validity and repeatability of the diagnostic tests used. When such data are available, for instance on carpal tunnel syndrome, they do not indicate that traditional eponymous tests are either highly specific or sensitive. ${ }^{4}$ One way of improving the reliability of clinical tests would be the production of a video or manual of test techniques showing details of their conduct. The use of electrophysiological measurements of nerve conduction is limited by the difficulty in establishing defined limits of normality.

It was noted that for most of the conditions considered during this exercise, there are no characteristic pathological or physiological changes that could be used as standards to validate tests for routine use in studies in the clinic and workplace. This absence of absolute measures of diagnostic accuracy makes for confusion in the assessment of validity. There is scope for modern imaging techniques such as MRI in validating tests more suitable for field investigation.

Knowledge of the natural history and prognosis of an upper limb disorder is usually taken into consideration during diagnosis. However, it was agreed that not enough is known about evolution of most of the disorders considered here for duration to be included in the diagnostic criteria. The exception was frozen shoulder, with its well defined sequence of symptoms. Similarly, measures of severity are not well defined. Agreed criteria for staging and severity would be an aid to better longitudinal studies.

Other constraints on validity concern the choice of participants and the Delphi exercise itself. Although the participants were all actively engaged in some aspect of work related upper limb disorders and were all considered to be experts in this field, such a small number could not be considered to be thoroughly representative of national opinion, let alone able to voice authoritative views on diagnostic differences at an international level.

The Delphi exercise is not perfect either. Although it is a useful tool for identifying consensus - and much in vogue at presentthe whole procedure could be construed as an attempt to squeeze quarts into pint pots. Its merit is that it does focus minds on achieving consensus but it leaves some issues, such as the extent and distribution of symptoms to be taken up and described by the researchers undertaking the studies. It is a positive step on the road to comparable studies and thus to the development of valid criteria for prevention. It is not an end in itself.
Within the constraints imposed by the lack of identifiable pathology, poor information on the sensitivity and specificity of various clinical and laboratory tests, and concerns over the validity of the Delphi process, this exercise produced clear and concise consensus case definitions for surveillance purposes for CTS, tenosynovitis of the wrist, de Quervain's disease of the wrist, epicondylitis, shoulder capsulitis (frozen shoulder), and shoulder tendonitis that were considered usable for a range of clinical and epidemiological investigations. These conditions are all associated with well defined anatomical entities even if the underlying pathology is not fully established. The condition that the consensus meeting labelled non-specific diffuse forearm pain differed in that no anatomical structure could be defined and that the diagnosis was by exclusion. As it had been decided at the onset of this exercise that inclusion of work relatedness in a case definition would cause difficulties in studies designed to establish work relatedness, the participants agreed that this should not be included here. It was recognised though that non-specific diffuse forearm pain might seem an unsatisfactory label for one case definition, it does reflect the current state of knowledge and accepts that forearm pain not associated with any specific anatomical structure may well be a real entity and is certainly associated with considerable morbidity. Further work is needed to characterise distinguishing features of this complaint and to see whether there are constellations of separable signs and symptoms for subsets of patients.

The workshop participants noted that the naming of upper limb disorders is chaotic: some are named by location, some by presumed cause, leading to incorrect attribution of blame. In several cases "-itis" is used for conditions which are not inflammatory. The workshop participants favoured pathological definitions when appropriate but sought to avoid false labels for non-specific conditions when observable pathology is indeterminate. Several important causes of upper limb pain were not discussed and require further consideration and the case definition for surveillance purposes. Foremost among these is pain referred from the neck. Referred cardiac and pleural pain also need consideration in any overall diagnostic framework.

The approach with a Delphi exercise followed by a consensus workshop among the professional groups involved in the epidemiology, diagnosis, and treatment of these disorders in the United Kingdom was seen as an effective means of securing agreed case definition criteria for surveillance purposes for several upper limb disorders. These "Birmingham criteria" will no doubt be refined as the results of more well designed studies become available. Use of the criteria developed at this meeting should help to ensure that future studies are comparable, that data sets can be combined across industries and even countries, and that time trends could be identified. ${ }^{3}$ If the criteria described here have to be modified in a specific study to take into account local circumstances, reference to them 
will allow diagnostic differences to be highlighted in subsequent reporting.

This study would not have been possible without the enthusiastic cooperation of the core participants in the Delphi exercise. tic cooperation of the core participants in the Delphi exercise. We thank A Auty, D M Baxendine, P Buckle, F Burke, D Cog-
gon, C Cooper, T Davis, A Dawson, M Doherty, I Felix, R

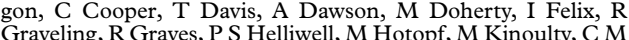
Graveling, R Graves, P S Helliwell, M Hotopf, M Kinoulty, C M Lambert, G Macfarlane, C Mackay, N Murray, J Osman, S
Robertson, L Rushton, A Silman, P Skew, D Wheeler, and N Williams for their professional input to this exercise. We thank Professor C Cooper, Mr T Davis, Professor M Doherty, Dr P S Helliwell, and Dr G MacFarlane for their help in the analysis and presentation of the Delphi data. We are indebted to Julie Tucker for her management of the various stages of the Delphi process. We also thank Dr R McCaig from the Health and Safety Executive, for his support and encouragement. Finally we are most grateful to the Health and Safety Executive for providing the sponsorship for this exercise.

1 Health and Safety Commission. Health and safety statistics. Statistical supplement to the 1994-5 annual report. Sudbury, Suffolk: HSE Books, 1995.

2 Hodgson JT, Jones JR, Elliot RC, et al. Self reported work related illness. Sudbury, Suffolk: HSE Books, 1993. (Research paper 33.)

3 Yassi A. Repetitive strain injuries. Lancet. 1997;349:943-7. 4 Hagberg M, Silverstein B, Wells R, et al. In: Kuorinka I, Forcier L, eds. Work related musculoskeletal disorders (WMSDS). A reference book for prevention. London: Taylor and Francis, 1995.
5 Harrington JM, Gompertz D, McCaig RH. Repetitive strain injuries. Lancet 1997;349:1701-2.

6 Sackett DL. Evaluation of clinical method. In: Weatherall DJ, Ledingham JGG, Warrell DA, eds. Oxford textbook of medicine. 3rd ed. Oxford: Oxford University Press, 1996:1521

7 Hagberg M, Wegman DH. Prevalence rates and odds ratios of shoulder-neck disease in different occupational groups. Br f Ind Med 1987;44:602-10.

8 Matte TD, Baker EL, Honchar PA. The selection and definition of targeted work-related conditions for surveillance under SENSOR. Am 7 Public Health 1989;79(suppl):21-5.

9 Helliwell PS. Diagnostic criteria for work related upper limb disorders. Br f Rheumatol 1996;35:1195-6.

10 Gemne G, Pyykko I, Taylor W, et al. The Stockholm workshop scale for the classification of cold-induced Raynaud's phenomena in the hand-arm vibration syndrome. Scand $\mathcal{F}$ Work Environ Health 1987;13:275-8.

11 Delbecq AL, Van de Ven AH, Gustafson DH. Group techniques for program planning. Illinois: Scott Foresman, 1975:83-107.

12 Rosenbaum RB, Ochoa JL. Carpal tunnel syndrome and other disorders of the median nerve. Oxford: Butterworth Heinemann, 1993.

3 Grundberg AB. Carpal tunnel decompression in spite of normal electromyography. F Hand Surg 1983;8:348-9.

14 Katz JN, Larson MG, Sabra A. The carpal tunnel syndrome: diagnostic utility of the history and physical examination findings. Ann Intern Med 1990;112:321-7.

15 Doherty M, Dacre J, Dieppe P, et al. The GALS locomotor screen. Diagnostic support aid for upper limb disorders. screen. Diagnostic support aid for

\section{Rejected manuscripts}

From February 1994, authors whose submitted articles are rejected will be advised of the decision and one copy of the article, together with any reviewer's comments, will be returned to them. The fournal will destroy remaining copies of the article but correspondence and reviewers' comments will be kept. 